\title{
ASYMPTOTICS FOR THE LENGTH OF A MINIMAL TRIANGULATION ON A RANDOM SAMPLE ${ }^{1}$
}

\author{
BY J. E. YuKICH \\ Lehigh University
}

Given $F \subset[0,1]^{2}$ and finite, let $\sigma(F)$ denote the length of the minimal Steiner triangulation of points in $F$. By showing that minimal Steiner triangulations fit into the theory of subadditive and superadditive Euclidean functionals, we prove under a mild regularity condition that

$$
\lim _{n \rightarrow \infty} \sigma\left(X_{1}, \ldots, X_{n}\right) / n^{1 / 2}=\beta \int_{[0,1]^{2}} f(x)^{1 / 2} d x \quad \text { c.c. }
$$

where $X_{1}, \ldots, X_{n}$ are i.i.d. random variables with values in $[0,1]^{2}, \beta$ is a positive constant, $f$ is the density of the absolutely continuous part of the law of $X_{1}$, and c.c. denotes complete convergence. This extends the work of Steele. The result extends naturally to dimension three and describes the asymptotics for the probabilistic Plateau functional, thus making progress on a question of Beardwood, Halton and Hammersley. Rates of convergence are also found.

1. Introduction. In their pioneering paper, Beardwood, Halton and Hammersley (1959) showed that the length of the shortest tour $T\left(X_{1}, \ldots, X_{n}\right)$ on independent and identically distributed (i.i.d.) random variables $X_{1}, \ldots, X_{n}$ with values in the unit square $[0,1]^{2}$ satisfies

$$
\lim _{n \rightarrow \infty} T\left(X_{1}, \ldots, X_{n}\right) / n^{1 / 2}=\beta \int_{[0,1]^{2}} f(x)^{1 / 2} d x \quad \text { a.s., }
$$

where $f$ is the density of the absolutely continuous part of $X_{1}$ and $\beta$ is a positive constant. This celebrated limit result for the traveling salesman problem (TSP) is one of the central theorems in the probability theory of combinatorial optimization. It is known that several other functionals in combinatorial optimization and operations research, including lengths of minimal matchings and minimal spanning trees, also satisfy the asymptotics (1.0). We refer to Rhee (1993), Steele (1981, 1997) and Redmond and Yukich (1994, 1996), and Yukich $(1995,1998)$ for details.

In this paper we develop the asymptotic behavior of the total edge length of the minimal triangulation on $n$ points which are independently and identically distributed on the unit square $[0,1]^{2}$. We show that modulo a constant factor the asymptotics are identical to those (1.0) of the TSP functional $T$.

\footnotetext{
Received April 1995; revised February 1998.

${ }^{1}$ Supported in part by NSA Grant MDA904-95-H-1005 and the U.S. Army Research Office through the Mathematical Sciences Institute of Cornell University.

AMS 1991 subject classifications. Primary 60F15, 60D05; secondary 68C05, 68E10.

Key words and phrases. Triangulation, subadditive and superadditive Euclidean functionals, traveling salesman problem, discrete probabilistic Plateau problem.
} 
This extends and generalizes earlier work of Steele (1982), who took the first important steps in the study of the length of random triangulations. We extend our results to three dimensions and in this way obtain asymptotics for the probabilistic Plateau functional.

Triangulations arise naturally in many areas of mathematics, including computational geometry. They have applications to surface interpolation, geometric searching techniques, and the finite-element method. See Preparata and Shamos (1985) and Bern and Eppstein (1992) for thorough treatments.

We now formulate the minimal triangulation problem precisely. Given a finite set $F$ of points in $[0,1]^{2}$, a triangulation of $F$ is a decomposition of $[0,1]^{2}$ into triangles whose vertices coincide with the set $F$ and the corners of $[0,1]^{2}$. In general, a set $F$ admits more than one triangulation. The total edge length of a triangulation is the sum of the lengths of the edges in the triangulation.

Given $1<\delta<\infty$, a " $\delta$-triangulation" of $F$ is a triangulation in which all triangles have aspect ratios which are uniformly bounded by $\delta$, that is the ratio of the radii of the circumscribed and inscribed balls is bounded uniformly by $\delta$ over all triangles. Such triangulations have a number of motivations and applications including some in computational learning theory [Salzberg, Delcher, Heath and Kasif (1991)]. Let $S_{\delta}$ denote a function which assigns to each set $F \subset[0,1]^{2}$ a $\delta$-triangulation of $F$ which has the least total edge length. Let $S_{\delta}(F)$ denote the graph of this triangulation (possibly empty, depending on the choice of $F$ and $\sigma$ ) and let $\left|S_{\delta}(F)\right|$ denote the total edge length of $S_{\sigma}(F)$. We will occasionally write $S(F)$ for $S_{\delta}(F)$.

If $F \subset[0,1]^{2}$ and $G \subset[0,1]^{2}$ is an additional set of points distinct from $F$ (i.e., a Steiner set) then $S_{\delta}(F \cup G)$ denotes the graph of a Steiner $\delta$-triangulation of $F$ with respect to $G$. We tacitly assume that $\delta>1$ is chosen large enough such that for every set $F$ there is a Steiner set $G$ for which $S_{\delta}(F \cup G)$ exists. We define the length of the minimal Steiner $\delta$-triangulation of $F$ by

$$
\sigma_{\delta}(F):=\inf _{G}\left|S_{\delta}(F \cup G)\right|,
$$

where $G$ ranges over all finite sets of Steiner points including the empty set. It is conceivable that adding more and more Steiner points may decrease the total length of the triangulation [see Bern and Eppstein (1992)]. Thus it is unclear whether the infimum is realized by a set $G$ and it is thus an open problem whether a "minimal $\delta$-triangulation" actually exists.

Without loss of generality we may assume that $G$ ranges over points in $[0,1]^{2}$ with rational coordinates. The set of admissible Steiner triangulations of a set $F$ thus has the cardinality of the countable set $\cup_{n=1}^{\infty}(\mathbb{Q} \times \mathbb{Q})^{n}$. This technical remark will ensure the measurability of $\sigma_{\delta}\left(X_{1}, \ldots, X_{n}\right)$, where $X_{i}$, $i \geq 1$, are random variables.

The main goals of this paper are to develop some basic deterministic properties of $\delta$-triangulations and to use these to determine the asymptotic behavior of $\sigma_{\delta}\left(X_{1}, \ldots, X_{n}\right)$, where $X_{i}, i \geq 1$, are i.i.d. random variables with values in the unit square. 
The minimal triangulation length $\sigma_{\delta}$ is an example of a Euclidean functional: it is a real-valued function defined on the finite subsets $F$ of $[0,1]^{2}$ with its value determined by the Euclidean distances between points in $F$. Up to now, the length $\sigma_{\delta}$ has not fit into any established theory of subadditive Euclidean functionals. This peculiarity has been noted by Steele (1982), who points out that $\sigma_{\delta}$ does not appear to enjoy the "simple subadditive" property

$$
\sigma_{\delta}(F \cup G) \leq \sigma_{\delta}(F)+\sigma_{\delta}(G)+C, \quad F, G \subset[0,1]^{2},
$$

where here and elsewhere $C:=C(\delta)$ is a constant depending only on $\delta$ and whose value may vary from line to line. One of the main points of this article is to show that the length $\sigma_{\delta}$ fits nicely within the theory of subadditive and superadditive Euclidean functionals [see Redmond and Yukich $(1994,1996)$ ]. In this way we readily show for each fixed $\delta, 1<\delta<\infty$, that $\sigma_{\delta}$ resembles the traveling salesman functional in that modulo constant factors, they have identical asymptotic properties.

To keep the presentation self-contained, we now recall the essentials of the theory of Euclidean functionals. We refer to Steele (1981, 1997), Rhee (1993), Redmond and Yukich (1994, 1996), and Yukich (1998) for additional background, details, and examples.

For both theoretical and practical purposes it is convenient to define Euclidean functionals on pairs $(F, R)$, where $R$ is a $d$-dimensional rectangle in $\mathbb{R}^{d}$ and $F$ is a finite subset of $\mathbb{R}^{d}$. We let $\mathscr{R}:=\mathscr{R}(d)$ denote the collection of $d$-dimensional rectangles in $\mathbb{R}^{d}$.

Given $0<p<d$, we say that $L^{p}$ is a continuous subadditive Euclidean functional of order $p$ if for all rectangles $R \in \mathscr{R}$ we have $L^{p}(\varnothing, R)=0$ and for all finite sets $F \subset \mathbb{R}^{d}$ we have the following conditions:

(1.1) (Homogeneity). $L^{p}(\alpha F, \alpha R)=\alpha^{p} L^{p}(F, R)$ for all $\alpha>0$;

(1.2) (Translation invariance). $L^{p}(y+F, y+R)=L^{p}(F, R)$ for all $y \in \mathbb{R}^{d}$;

(1.3) (Geometric subadditivity). There exists a constant $C_{1}$ such that for all positive integers $m$ and all subsets $F$ of $[0,1]^{d}$,

$$
L^{p}\left(F,[0,1]^{d}\right) \leq \sum_{i=1}^{m^{d}} L^{p}\left(F \cap Q_{i}, Q_{i}\right)+C_{1} m^{d-p},
$$

where $\left(Q_{i}\right)_{i \leq m^{d}}$ is the partition of $[0,1]^{d}$ into $m^{d}$ subcubes of edge length $m^{-1}$

(1.4) (Continuity). There exists a constant $C_{2}$ such that for all finite subsets $F$ and $G$ of $[0,1]^{d}$,

$$
\left|L^{p}\left(F \cup G,[0,1]^{d}\right)-L^{p}\left(F,[0,1]^{d}\right)\right| \leq C_{2}\{\operatorname{card} G\}^{(d-p) / d} .
$$

Superadditive Euclidean functionals are defined in a way that naturally parallels the conditions (1.1)-(1.4). Say that $L^{p}$ is a continuous superadditive 
Euclidean functional of order $p, 0<p<d$, if (1.1), (1.2), (1.4) and the following hold:

(1.3') (Geometric superadditivity). There exists a constant $C_{1}^{\prime}$ such that for all positive integers $m$ and subsets $F$ of $[0,1]^{d}$,

$$
L^{p}\left(F,[0,1]^{d}\right) \geq \sum_{i=1}^{m^{d}} L^{p}\left(F \cap Q_{i}, Q_{i}\right)-C_{1}^{\prime} m^{d-p},
$$

where $\left(Q_{i}\right)_{i \leq m^{d}}$ is the partition given in (1.3).

A subadditive Euclidean functional $L^{p}$ of order $p$ is quasi-additive if there exists a superadditive Euclidean functional $L_{r}^{p}$ of order $p$ such that $L_{r}^{p} \leq L^{p}$ and $L_{r}^{p}$ closely approximates $L^{p}$ on homogeneous samples, that is

$$
\begin{aligned}
& \left|\mathbb{E} L^{p}\left(\left\{U_{1}, \ldots, U_{n}\right\},[0,1]^{d}\right)-\mathbb{E} L_{r}^{p}\left(\left\{U_{1}, \ldots, U_{n}\right\},[0,1]^{d}\right)\right| \\
& \quad=o\left(n^{(d-p) / d}\right) .
\end{aligned}
$$

Here and elsewhere $U_{i}, i \geq 1$, are i.i.d. uniform random variables on $[0,1]^{d}$. As shown in Redmond and Yukich (1994, 1996), quasi-additive functionals form a large class and include the traveling salesman, minimal spanning tree, minimal matching, and semi-matching functionals. The superadditive functional $L_{r}^{p}$ is usually a boundary rooted version of $L^{p}$ in the sense that edges may be rooted to the boundary of $[0,1]^{d}$ and subsequent travel on the boundary is free.

Quasi-additive functionals enjoy growth rates in the complete convergence (c.c.) sense. Recall that $Y_{n} \rightarrow Y$ in the sense of complete convergence iff for all $\varepsilon>0, \sum_{n=1}^{\infty} P\left\{\left|Y_{n}-Y\right|>\varepsilon\right\}<\infty$. Quasi-additive functionals have pleasing asymptotics and satisfy the following general result [cf. Redmond and Yukich $(1994,1996)]$.

THEOREM 1.1 (Umbrella theorem for Euclidean functionals). Let $L^{p}$ and $L_{r}^{p}$ be continuous subadditive and superadditive Euclidean functionals of order $p, 0<p<d$, respectively. If $L^{p}$ and $L_{r}^{p}$ satisfy the approximation (1.5), $L_{r}^{p} \leq L^{p}$, and $X_{i}, i \geq 1$, are i.i.d. random variables with values in $[0,1]^{d}$, then

$$
\begin{aligned}
\lim _{n \rightarrow \infty} L^{p}\left(\left\{X_{1}, \ldots, X_{n}\right\},[0,1]^{d}\right) / n^{(d-p) / d} \\
\quad=\beta\left(L^{p}, d\right) \int_{[0,1]^{d}} f(x)^{(d-p) / d} d x \quad c . c .,
\end{aligned}
$$

where $\beta\left(L^{p}, d\right)$ is a positive constant and $f$ is the density of the absolutely continuous part of the law of $X_{1}$.

It has been long expected that for each fixed $1<\delta<\infty$ the minimal triangulation length $\sigma_{\delta}$ conforms to the asymptotics (1.6), but the proof has remained elusive. This is due largely to the fact that $\sigma_{\delta}$ lacks an easily established "simple subadditivity" property and that not much is known about $\sigma_{\delta}$. One of the main contributions of this paper is develop some basic properties of stochastic triangulations and in this way show that $\sigma_{\delta}$ does satisfy (1.6). This is accomplished by demonstrating that triangulations fit 
naturally within the framework of Theorem 1.1, which is more encompassing than one might guess. Showing that triangulations conform to the conditions of Theorem 1.1 involves methods combining probability and geometry. The arguments depend upon a judicious definition of a superadditive "boundary triangulation functional." By verifying that subadditive triangulations and superadditive boundary triangulations satisfy the conditions of Theorem 1.1 when $p=1$ and $d=2$, we may prove our first main result, which makes progress on a problem raised by Steele (1982) and which shows that minimal triangulations resemble minimal tours.

THEOREM 1.2 (Asymptotics for minimal triangulations). Let $X_{i}, i \geq 1$, be i.i.d. random variables with values in $[0,1]^{2}$. Fix $1<\delta<\infty$ and consider the minimal triangulation length $\sigma_{\delta}$. Then

$$
\lim _{n \rightarrow \infty} \sigma_{\delta}\left(X_{1}, \ldots, X_{n}\right) / n^{1 / 2}=\beta\left(\sigma_{\delta}\right) \int_{[0,1]^{2}} f(x)^{1 / 2} d x \quad \text { c.c. },
$$

where $\beta\left(\sigma_{\delta}\right)$ is a positive constant and $f$ denotes the density of the absolutely continuous part of the law of $X_{1}$.

The above result adds to the work of Steele (1982), who considers the case $\delta=\infty$, that is, the case involving no restrictions on the aspect ratios of the triangles. Steele uses geometric subadditivity of triangulations to establish (1.7) for $\sigma_{\infty}$ in the special case that $X_{1}, \ldots, X_{n}$ are uniformly distributed random variables on $[0,1]^{2}$. According to Steele (1982), Theorem 1.2 addresses a problem of György Turán.

By placing the triangulation functional in the context of quasi-additive functionals, we may, moreover, derive rates of convergence in a natural way.

Theorem 1.3 (Rates of convergence). Fix $1<\delta<\infty$. The mean of $\sigma_{\delta}$ on uniform samples in $[0,1]^{2}$ satisfies

$$
\left|\mathbb{E} \sigma_{\delta}\left(U_{1}, \ldots, U_{n}\right)-\beta\left(\sigma_{\delta}\right) n^{1 / 2}\right| \leq C(n \log n)^{1 / 4}
$$

The above two-dimensional results have a natural three-dimensional ana$\log$. Given a finite set $F$ of points in $[0,1]^{3}$, a tetrahedralization of $F$ is a decomposition of $[0,1]^{3}$ into tetrahedra whose vertices coincide with the points in $F$ and the corners of the cube $[0,1]^{3}$. In general, $F$ admits more than one tetrahedralization. The total surface area of a tetrahedralization is the sum of the areas of the triangular faces.

Given $1<D<\infty$, a $D$-tetrahedralization is one in which the tetrahedra have aspect ratios uniformly bounded by $D$, that is the ratio of the radii of the circumscribed and inscribed spheres is bounded uniformly by $D$ over all tetrahedra. This regularity condition insures that the cube of the length of a tetrahedral edge is bounded by a constant multiple of the volume of the tetrahedron, a fact which will be useful in the sequel.

Given $1<D<\infty$, let $T_{D}$ denote a function which assigns to each set $F \subset[0,1]^{3}$ a $D$-tetrahedralization of $F$ having the least total surface area. Let $T_{D}(F)$ denote the graph of the tetrahedralization and let its total surface 
area be denoted by $\left|T_{D}(F)\right|$. If $G \subset[0,1]^{3}$ is a set of Steiner points, then we let $T_{D}(F \cup G)$ denote the graph of the corresponding Steiner $D$-tetrahedralization of $F$. We tacitly assume that $D>1$ is chosen large enough so that for every set $F \subset[0,1]^{3}$ there is a Steiner set $G$ for which $T_{D}(F \cup G)$ exists. Analogously to $\sigma_{\delta}(F)$, define the area $\tau_{D}(F)$ of the minimal Steiner $D$-tetrahedralization of $F$ by

$$
\tau_{D}(F):=\inf _{G}\left|T_{D}(F \cup G)\right|,
$$

where $G$ ranges over all finite sets of Steiner points. Here $\tau_{D}(F)$ may be thought of as the discrete Plateau functional for the point set $F$.

As in the definition of $\sigma_{\delta}$, we may without loss of generality restrict attention to Steiner points with rational coordinates. In this way we ensure the measurability of $\tau_{D}\left(X_{1}, \ldots, X_{n}\right)$, where $X_{i}, i \geq 1$, are random variables.

In Section 6 we will prove the following analog of Theorem 1.2. This makes progress on a question raised by Beardwood, Halton and Hammersley (1959).

THEOREM 1.4 (Asymptotics for minimal tetrahedralizations). Let $X_{i}, i \geq 1$, be i.i.d. random variables with values in $[0,1]^{3}$. Then for each fixed $1<D<\infty$ we have

$$
\lim _{n \rightarrow \infty} \tau_{D}\left(X_{1}, \ldots, X_{n}\right) / n^{1 / 3}=\beta\left(\tau_{D}\right) \int_{[0,1]^{3}} f(x)^{1 / 3} d x \quad c . c .,
$$

where $\beta\left(\tau_{D}\right)$ is a positive constant and f denotes the density of the absolutely continuous part of the law of $X_{1}$.

We anticipate that Theorems 1.3 and 1.4 admit extensions to higher dimensions. In this way we could perhaps find asymptotics for the randomized version of the problem of Douglas, which considers minimal surfaces in higher dimensions. Beardwood, Halton, and Hammersley (1959) were apparently the first to consider such a problem. In their admittedly cryptic remarks, they recognize the potential applicability of subadditivity methods but do not develop the necessary mathematics. For a treatment of the problem of Douglas we refer to Chapter 4 of Courant and Schiffer (1950) and Douglas (1939). We will not pursue generalizations to higher dimensions here.

We summarize the contributions of this paper.

1. Modulo constant factors, for each $1<\delta<\infty$ the triangulation length $\sigma_{\delta}$ is shown to have an asymptotic behavior identical to that of the TSP, making progress on a problem of Steele (1982).

2. The collection of quasi-additive Euclidean functionals, which includes the TSP, minimal spanning tree, minimal matching and semi-matching functionals, is shown to include the minimal triangulation and discrete Plateau functionals as well.

3. A rate of convergence for the mean of $\sigma_{\delta}\left(U_{1}, \ldots, U_{n}\right), 1<\delta<\infty$, is obtained.

4. Asymptotics for the discrete probabilistic Plateau functional $\tau_{D}, 1<D<\infty$, are found. 
This paper is organized as follows. Section 2 presents the boundary triangulation functional, which forms the cornerstone of the theory. Sections 3 and 4 show that the minimal triangulation length and its superadditive boundary version are continuous Euclidean functionals on $\mathbb{R}^{2}$ of order 1 , that is, satisfy conditions (1.1)-(1.4) and (1.1), (1.2), (1.3'), (1.4), respectively, with $p=1$ and $d=2$. Finally, Section 5 proves that the minimal triangulation length and its boundary version satisfy the closeness condition (1.5). In this way we prove Theorem 1.2. Section 6 proves Theorem 1.4.

To facilitate the exposition and lighten the notation, we will often omit mention of " $\delta$ " and " $D$ " when referring to $\delta$-triangulations and $D$-tetrahedralizations, respectively. Moreover, when it is clear from the context, we will often simply write $\sigma$ for $\sigma_{\delta}$ and $\tau$ for $\tau_{D}$.

2. The boundary triangulation functional. In earlier work on Euclidean functionals initiated by Redmond and Yukich $(1994,1996)$, it was recognized that the "boundary functional" $L_{r}$ associated with an optimization problem $L$ plays a key role in simplifying both the conceptual and technical analysis. Roughly speaking, boundary functionals $L_{r}$ are defined on subsets of a rectangle $R$ and differ from the functional $L$ only in that edges on the boundary of $R$ are assigned zero length. In the case of the minimal tour functional, this means that travel along the boundary is effectively free. The purpose of this section is to define appropriately the "boundary $\delta$-triangulation functional," where throughout $1<\delta<\infty$ is arbitrary but fixed.

Given a convex polygon $\Delta \subset[0,1]^{2}$ and $F \subset \mathbb{R}^{2}$ whose convex hull co( $\left.F\right)$ contains $\Delta$, consider a $\delta$-triangulation of $\operatorname{co}(F)$. Such a $\delta$-triangulation partitions $\operatorname{co}(F)$ into triangles whose vertices coincide with $F$ and whose aspect ratios are bounded by $\delta$. The $\delta$-triangulation of $\operatorname{co}(F)$, which has the property that the total edge length of the restriction of its graph to the interior of $\Delta$ is minimal is called the "boundary $\delta$-triangulation" of $\Delta$ induced by $F$ and is denoted by $S_{r, \delta}(F, \Delta)$. A boundary $\delta$-triangulation of $[0,1]^{2}$ thus generates a partition of $[0,1]^{2}$ consisting of the usual triangles as well as perhaps quadrilaterals, pentagons and even hexagons. When the context is clear, we will omit mention of $\delta$ and refer to boundary $\delta$-triangulations as simply boundary triangulations.

By the "total edge length" $\left|S_{r, \delta}(F, \Delta)\right|$ of the boundary $\delta$-triangulation $S_{r, \delta}(F, \Delta)$ we mean the sum of the lengths of the edges in the triangulation which lie in the interior of $\Delta$. Analogously to $\sigma(F):=\sigma_{\delta}(F)$, define for all $F \subset \Delta$ the length of the "minimal boundary $\delta$-triangulation" of $F$ with respect to $\Delta$ by

$$
\sigma_{r, \delta}(F, \Delta):=\inf _{G}\left|S_{r, \delta}(F \cup G, \Delta)\right|,
$$

where $G$ ranges over finite sets of Steiner points in $\mathbb{R}^{2}$ with the property that the convex hull of $F \cup G$ contains $\Delta$. Without loss of generality we will assume that the points in $G$ have rational coordinates. Minimal boundary triangulations may fail to exist for the same reasons that the standard 
minimal triangulation may not exist. We will occasionally condense notation and write $\sigma_{r}$ for $\sigma_{r, \delta}$ and $S_{r}$ for $S_{r, \delta}$. It is clear from the definitions that $\sigma_{r}\left(F,[0,1]^{2}\right) \leq \sigma(F)$ for all $F \subset[0,1]^{2}$.

The study of the minimal boundary triangulation length $\sigma_{r}$ is motivated by the fact that it enjoys geometric superadditivity: for all $F \subset[0,1]^{2}$ we have

$$
\sigma_{r}\left(F,[0,1]^{2}\right) \geq \sum_{i=1}^{m^{2}} \sigma_{r}\left(F \cap Q_{i}, Q_{i}\right) .
$$

To see this, find a sequence $G_{n}$ of Steiner sets such that the boundary triangulations $S_{r}\left(F \cup G_{n},[0,1]^{2}\right), n \geq 1$, have lengths $\left|S_{r}\left(F \cup G_{n},[0,1]^{2}\right)\right|$, decreasing down to $\sigma_{r}\left(F,[0,1]^{2}\right)$ as $n \rightarrow \infty$. For each $1 \leq i \leq m^{2}$, let $S_{r}^{i}(F \cup$ $\left.G_{n},[0,1]^{2}\right)$ denote the boundary triangulation of $Q_{i}$ generated by the intersection of $S_{r}\left(F \cup G_{n},[0,1]^{2}\right)$ with subsquare $Q_{i}$. Then for each $n \geq 1$ we have

$$
\left|S_{r}\left(F \cup G_{n},[0,1]^{2}\right)\right|=\sum_{i=1}^{m^{2}}\left|S_{r}^{i}\left(F \cup G_{n},[0,1]^{2}\right)\right| \geq \sum_{i=1}^{m^{2}} \sigma_{r}\left(F \cap Q_{i}, Q_{i}\right),
$$

where the inequality follows by minimality of $\sigma_{r}$. Let $n$ tend to infinity to deduce superadditivity (2.1). Summarizing, we have shown the following lemma.

Lemma 2.1. Here $\sigma_{r}$ is superadditive and satisfies $\left(1.3^{\prime}\right)$ with $C_{1}^{\prime}=0$.

Having defined the minimal triangulation lengths $\sigma$ and $\sigma_{r}$ we are positioned to prove Theorem 1.2. We must verify that $\sigma$ and $\sigma_{r}$ are continuous subadditive and superadditive Euclidean functionals of order 1, respectively, and that they satisfy the closeness condition (1.5) with $p=1$ and $d=2$. This is shown in the following three sections.

3. Minimal triangulations are subadditive and continuous. We will verify that the length $\sigma:=\sigma_{\delta}$ of the minimal triangulation satisfies conditions (1.1)-(1.4) with $p=1$ and $d=2$. Throughout, let $1<\delta<\infty$ be arbitrary but fixed and write $\sigma$ for $\sigma_{\delta}$. In the sequel we show that the boundary triangulation length $\sigma_{r}:=\sigma_{r, \delta}$ satisfies (1.1), (1.2), (1.3') and (1.4) with $p=1$ and $d=2$.

It will be helpful to consider the triangulation of sets in regions other than the unit square. We thus enlarge the definition of triangulations in the following way.

Definition 3.1. Let $F \subset \Delta$ be a finite set, where $\Delta \subset[0,1]^{2}$ is a convex polygon. Consider all Steiner $\delta$-triangulations $S_{\delta}(F \cup G, \Delta), G \subset \Delta$, of $\Delta$, that is, all decompositions of $\Delta$ into triangles whose vertices coincide with $F \cup G$ and the corners of $\Delta$ and whose aspect ratios are bounded by $\delta$. Let $S(F \cup$ $G, \Delta)$ have total edge length $\left|S_{\delta}(F \cup G, \Delta)\right|$. Define

$$
\sigma_{\delta}(F, \Delta):=\inf _{G}\left|S_{\delta}(F \cup G, \Delta)\right|,
$$


where $G$ ranges over all finite sets in $\Delta$. We call $\sigma(F, \Delta):=\sigma_{\delta}(F, \Delta)$ the length of the minimal $\delta$-triangulation of $F$ with respect to $\Delta$. When $\Delta=[0,1]^{2}$, we will simply write $\sigma(F)$ for $\sigma\left(F,[0,1]^{2}\right)$.

Notice that $\sigma$, considered as a function on pairs $(F, R)$, satisfies homogeneity (1.1) and translation invariance (1.2) with $p=1 ; \sigma$ also satisfies geometric subadditivity (1.3) with no error term.

LemMa 3.2. For every $F \subset[0,1]^{2}$ we have

$$
\sigma(F) \leq \sum_{i=1}^{m^{2}} \sigma\left(F \cap Q_{i}, Q_{i}\right),
$$

where $\left(Q_{i}\right)_{i \leq m^{2}}$ denotes the usual partition of $[0,1]^{2}$ into subsquares of edge length $\mathrm{m}^{-1}$.

Proof. For each $1 \leq i \leq m^{2}$, we may find a sequence of Steiner sets $G_{n}:=G_{n, i} \subset Q_{i}, n \geq 1$, such that the triangulations $S_{\delta}\left(\left(F \cup G_{n}\right) \cap Q_{i}, Q_{i}\right)$, $n \geq 1$, have lengths $\left|S_{\delta}\left(\left(F \cup G_{n}\right) \cap Q_{i}, Q_{i}\right)\right|$ which decrease down to the length of the minimal triangulation $\sigma\left(F \cap Q_{i}, Q_{i}\right)$ as $n$ tends to infinity. For each $n \geq 1$, the union of the local triangulations $S_{\delta}\left(\left(F \cup G_{n}\right) \cap Q_{i}, Q_{i}\right), 1 \leq i \leq m^{2}$, is a feasible Steiner triangulation of $[0,1]^{2}$. Minimality implies that for all $n \geq 1$,

$$
\sigma(F) \leq \sum_{i=1}^{m^{2}}\left|S_{\delta}\left(\left(F \cup G_{n}\right) \cap Q_{i}, Q_{i}\right)\right| .
$$

Now let $n$ tend to infinity to deduce (3.1).

When $Q_{i}$ and $Q_{j}$ are adjacent subsquares, the Steiner points on the boundary of $Q_{i}$ need not coincide with the Steiner points on the boundary of $Q_{j}$. We notice therefore that (3.1) would fail if we restricted attention to triangulations which were simplicial complexes.

By using geometric subadditivity (3.1) and following an induction argument due to Steele (1982), we obtain a useful growth bound for $\sigma$.

LEMMA 3.3. There is a finite constant $C$ such that for all $F \subset[0,1]^{2}$,

$$
\sigma(F) \leq C\{\operatorname{card} F\}^{1 / 2} \text {. }
$$

Proof [Steele (1982), Lemma 4.1]. For $n \geq 1$ set $\varphi(n):=\max \sigma(F)$, where the max is over all choices of $F \subset[0,1]^{2}$ with card $F=n$. Set $\varphi(0)=4$. Observe that by decomposing $[0,1]^{2}$ into four subsquares we obtain via Lemma 3.2,

$$
\varphi(n) \leq \max \left\{\frac{1}{2} \sum_{i=1}^{4} \varphi\left(a_{i}\right): \sum_{i=1}^{4} a_{i}=n\right\},
$$

where $1 / 2$ represents the scaling factor. By induction and the inequality $\frac{1}{2} \sum_{i=1}^{4} a_{i}^{1 / 2} \leq n^{1 / 2}$, it follows that $\varphi(n) \leq \mathrm{Cn}^{1 / 2}$. 
Notice that we may obtain growth bounds for $\sigma_{\delta}(F, \Delta), \Delta \subset[0,1]^{2}$ a convex polygon, by approximating $\Delta$ by the union of inscribed subsquares and applying growth bounds on the individual subsquares. This argument shows that there is a finite constant $C$ such that for all convex polygons $\Delta \subset[0,1]^{2}$ and all nonempty sets $F \subset \Delta$, we have $\sigma_{\delta}(F, \Delta) \leq C\{\operatorname{card} F\}^{1 / 2}$.

Finally, we may verify that $\sigma$ satisfies continuity (1.4) with $p=1$; namely, we show for all $F, G \subset[0,1]^{2}$ that

$$
|\sigma(F \cup G)-\sigma(F)| \leq C\{\operatorname{card} G\}^{1 / 2} .
$$

For all $\varepsilon>0$ and all $F \subset[0,1]^{2}$, let $G_{\varepsilon}:=G_{\varepsilon}(F)$ be the uniquely defined Steiner sets such that

$$
\left|S_{\delta}\left(F \cup G_{\varepsilon}\right)\right| \leq \sigma_{\delta}(F)+\varepsilon .
$$

Here we choose $G_{\varepsilon}(F)$ according to some specified algorithm; the exact choice of algorithm is not important. Here and henceforth, let $\Delta^{\varepsilon}(F)$ denote the collection of triangles defined by the triangulation $S_{\delta}\left(F \cup G_{\varepsilon}\right)$ and let $\mathscr{E}^{\varepsilon}:=$ $\mathscr{E}^{\varepsilon}(F)$ denote the collection of edges of these triangles. By assumption, the aspect ratios of the triangles in $\Delta^{\varepsilon}(F)$ are uniformly bounded by $\delta$. It follows that the square of the length of an edge of a triangle is bounded by a constant multiple of the triangular area. Since the sum of the areas of the triangles in $\Delta^{\varepsilon}(F)$ is just the area of the unit square, it follows that

$$
\sum_{E \in \mathscr{C}^{E}}|E|^{2} \leq C
$$

for some universal constant $C:=C(\delta)$ which does not depend on $\varepsilon$. Here and elsewhere, $|E|$ denotes the Euclidean length of the edge $E$.

To show continuity (3.3), it suffices by (3.5) to show

$$
\sigma(F) \leq \sigma(F \cup G) \leq \sigma(F)+C\left(\sum_{E \in \mathscr{C}^{\varepsilon}}|E|^{2}\right)^{1 / 2}\{\operatorname{card} G\}^{1 / 2} .
$$

Notice that the first inequality in (3.6) is a consequence of the intrinsic monotonicity of $\sigma$. To show (3.3), it thus suffices to prove the second inequality in (3.6).

Given $G$, we may assume that $G \cap F=\varnothing$. The points in $G$ are located in triangles $\Delta_{1}, \ldots, \Delta_{J}, J:=J(G)<\infty$, belonging to $\Delta^{\varepsilon}(F)$ [if a point in $G$ lies on an edge in $\mathscr{E}^{\varepsilon}(F)$, then it belongs to two triangles in $\Delta^{\varepsilon}(F)$ ]. Let $E_{i}$, $1 \leq i \leq J$, be the longest edge of triangle $\Delta_{i}, 1 \leq i \leq J$.

Observe that $\sigma(F \cup G)$ is bounded by $\left|S_{\delta}\left(F \cup G_{\varepsilon}\right)\right|$ and the sum of the lengths of the minimal triangulations of $G \cap \Delta_{i}$ with respect to $\Delta_{i}, 1 \leq i \leq J$. In other words,

$$
\begin{aligned}
\sigma(F \cup G) & \leq\left|S_{\delta}\left(F \cup G_{\varepsilon}\right)\right|+\sum_{i=1}^{J} \sigma\left(G \cap \Delta_{i}, \Delta_{i}\right) \\
& \leq\left|S_{\delta}\left(F \cup G_{\varepsilon}\right)\right|+C \sum_{i=1}^{J}\left|E_{i}\right|\left\{\operatorname{card}\left(G \cap \Delta_{i}\right)\right\}^{1 / 2}
\end{aligned}
$$


by scaling (1.1) and Lemma 3.3. Applying Hölder's inequality, we obtain

$$
\sigma(F \cup G) \leq\left|S_{\delta}\left(F \cup G_{\varepsilon}\right)\right|+C\left(\sum_{E \in \mathscr{E}_{\varepsilon}}|E|^{2}\right)^{1 / 2}\{\operatorname{card} G\}^{1 / 2},
$$

which together with (3.4) and (3.5), gives continuity (3.3) as desired.

4. Boundary minimal triangulations are superadditive and continuous. By Lemma 2.1 we know that the boundary triangulation functional $\sigma_{r}:=\sigma_{r, \delta}$ is superadditive $\left(1.3^{\prime}\right)$, where $1<\delta<\infty$ is arbitrary but fixed. It is also clear that $\sigma_{r}$ satisfies homogeneity (1.1) and is translation invariant (1.2) when $p=1$. It remains to verify continuity (1.4) which, when $p=1$ and $d=2$ requires showing for all $F, G \subset[0,1]^{2}$,

$$
\left|\sigma_{r}\left(F \cup G,[0,1]^{2}\right)-\sigma_{r}\left(F,[0,1]^{2}\right)\right| \leq C_{2}^{\prime}\{\operatorname{card} G\}^{1 / 2} .
$$

This will follow from a slight modification of the proof of the continuity of $\sigma$.

We first clarify the terminology. Let $1<\delta<\infty$ be arbitrary but fixed. As before, for all $\varepsilon>0$ and all $F \subset[0,1]^{2}$, let $G_{\varepsilon}:=G_{\varepsilon}(F)$ be the uniquely defined Steiner sets such that

$$
\left|S_{r}\left(F \cup G_{\varepsilon}\right)\right| \leq \sigma_{r}(F)+\varepsilon .
$$

Without loss of generality we may assume that $\operatorname{co}\left(F \cup G_{\varepsilon}\right)$ is contained in a large square $Q \subseteq[0,1]^{2}$ where the edge length of $Q$ is at most $C:=C(\delta)$.

Let $\Delta_{r}^{\varepsilon}(F)$ denote the collection of polygons formed by the boundary triangulation $S_{r}\left(F \cup G_{\varepsilon}\right)$. Let $\mathscr{E}_{r}^{\varepsilon}:=\mathscr{E}_{r}^{\varepsilon}(F)$ denote the set of all edges of these polygons. Since these edges form a subset of the edges of triangles contained in the square $Q$, it follows as in (3.5) that the sum of the squares of their lengths is bounded by a constant multiple of the area of $Q$, that is,

$$
\sum_{E \in \mathscr{E}_{r}^{\varepsilon}}|E|^{2} \leq C
$$

where $C:=C(\delta)$. If $\Delta$ is a polygon in $\Delta_{r}^{\varepsilon}(F)$ with diameter $D$ and if $G$ is a set of points in $\Delta$ then $\sigma(G, \Delta)$ is bounded by $C \cdot D \cdot\{\text { card } G\}^{1 / 2}$. The diameter $D$ is bounded by the sum of the lengths of the edges of $\Delta$ and $D^{2}$ is bounded by a constant multiple of the sum of the squares of the lengths of the edges of $\Delta$. The proof of continuity (4.1) follows exactly as in the proof of continuity (3.3) of $\sigma$.

5. Quasi-additivity. We have now verified that the minimal triangulation lengths $\sigma$ and $\sigma_{r}$ are continuous subadditive and superadditive Euclidean functionals of order 1 , respectively. We conclude the proof of Theorem 1.2 by showing quasi-additivity (1.5) of $\sigma:=\sigma_{\delta}$ and $\sigma_{r}:=\sigma_{r, \delta}$, where $1<\delta<\infty$ is arbitrary but fixed. We will actually establish the stronger bound

$$
\left|\mathbb{E} \sigma\left(U_{1}, \ldots, U_{n}\right)-\mathbb{E} \sigma_{r}\left(U_{1}, \ldots, U_{n}\right)\right| \leq C(n \log n)^{1 / 4},
$$

which will be useful in obtaining the rate (1.8). 
Given $\varepsilon>0$ and the random variables $U_{1}, \ldots, U_{n}$ we recall that $G_{\varepsilon}:=$ $G_{\varepsilon}\left(U_{1}, \ldots, U_{n}\right)$ are the uniquely defined Steiner sets with the property that

$$
\left|S_{r}\left(\left\{U_{1}, \ldots, U_{n}\right\} \cup G_{\varepsilon}\right)\right| \leq \sigma_{r}\left(U_{1}, \ldots, U_{n}\right)+\varepsilon .
$$

Let $\Delta_{r}^{\varepsilon}\left(U_{1}, \ldots, U_{n}\right)$ denote the collection of polygons generated by the boundary triangulation $S_{r}\left(\left\{U_{1}, \ldots, U_{n}\right) \cup G_{\varepsilon}\right)$ and let $\mathscr{E}^{\varepsilon}\left(U_{1}, \ldots, U_{n}\right)$ be the collection of polygonal edges formed from the intersection of $\Delta_{r}^{\varepsilon}\left(U_{1}, \ldots, U_{n}\right)$ and the interior of $[0,1]^{2}$. To prove (5.1) we first establish bounds on the lengths of the edges in $\mathscr{E}^{\varepsilon}\left(U_{1}, \ldots, U_{n}\right)$. This edge length bound implies that with high probability the collection $\Delta_{r}^{\varepsilon}\left(U_{1}, \ldots, U_{n}\right)$ contains neither hexagons nor pentagons with a side linking opposite sides of $[0,1]^{2}$. Thus with high probability there are at most four pentagons in $\Delta_{r}^{\varepsilon}\left(U_{1}, \ldots, U_{n}\right)$.

LEMMA 5.1. With high probability, all polygonal edges $E \in \mathscr{E}^{\varepsilon}\left(U_{1}, \ldots, U_{n}\right)$ have an edge length $|E|$ satisfying

$$
|E| \leq C(\log n / n)^{1 / 2} .
$$

REMARK. The precise meaning of the high probability statement (5.2) is as follows: for any prescribed $\alpha>0$ we can find $C:=C(\alpha)>0$ and a set $\Omega_{0}$ with $\operatorname{Pr}\left\{\Omega_{0}^{c}\right\}=O\left(n^{-\alpha}\right)$ such that on $\Omega_{0}$ all edges $E \in \mathscr{E}^{\varepsilon}\left(U_{1}, \ldots, U_{n}\right)$ satisfy the bound

$$
|E| \leq C(\log n / n)^{1 / 2}
$$

Proof. The proof is a simple consequence of the fact that the aspect ratios of the polygons in $\Delta_{r}^{\varepsilon}\left(U_{1}, \ldots, U_{n}\right)$ are bounded and therefore if an edge $E$ belongs to $\mathscr{E}^{\varepsilon}\left(U_{1}, \ldots, U_{n}\right)$, then there is a circle of radius $C|E|$ which is contained in $[0,1]^{2}$ and which does not contain any sample points, where $C:=C(\delta)$ is a constant depending only on $\delta$.

Indeed, for all $x \in[0,1]^{2}$ and $r>0$, let $B(x, r)$ designate the ball centered at $x$ with radius $r$ and let $E_{n}(r)$ denote the event that there is an edge $E \in \mathscr{E}^{\varepsilon}\left(U_{1}, \ldots, U_{n}\right)$ whose length exceeds $r$. Given $E_{n}(r)$, the bounded aspect ratio assumption implies the existence of a ball of radius at least $C r$ which is contained entirely within a polygon and thus does not contain any sample points. This is clearly true for edges $E$ which do not meet the boundary. For edges $E$ meeting the boundary there are several cases which may be checked in a straightforward fashion. In any case, there is a $C<\infty$ such that

$$
E_{n}(r) \subset\left\{\exists x \in[0,1]^{2}: B(x, C r) \subset[0,1]^{2}, B(x, C r) \cap\left\{U_{i}\right\}_{i \leq n}=\varnothing\right\} .
$$

Thus, $\operatorname{Pr}\left\{E_{n}(r)\right\}$ is bounded by the probability that there is "hole" of radius at least $\mathrm{Cr}$ in the sample $\left\{U_{1}, \ldots, U_{n}\right\}$. It is well known and easy to show that with high probability, holes with radius larger than $C(\log n / n)^{1 / 2}$ do not exist. Thus, with high probability, edges in $\mathscr{E}^{\varepsilon}\left(U_{1}, \ldots, U_{n}\right)$ have length less than $C(\log n / n)^{1 / 2}$. 
We require one more auxiliary result before proving (5.1). To simplify the notation, write $\sigma(n)$ for $\sigma\left(U_{1}, \ldots, U_{n}\right)$ and likewise for $\sigma_{r}(n)$, and $\mathscr{E}^{\varepsilon}(n)$. Let $S_{r}^{\varepsilon}(n):=S_{r}\left(\left\{U_{1}, \ldots, U_{n}\right\} \cup G_{\varepsilon}\right)$. Consider the edges in $\mathscr{E}^{\varepsilon}(n)$ which meet the boundary of $[0,1]^{2}$ and let $\Sigma^{\varepsilon}(n):=\Sigma^{\varepsilon}\left(U_{1}, \ldots, U_{n}\right)$ denote the sum of the lengths of these edges. The following lemma gives a crude yet sufficient upper bound for $\Sigma^{\varepsilon}(n)$.

Lemma 5.2. For all $0<\varepsilon<1, \quad \mathbb{E} \Sigma^{\varepsilon}(n) \leq C(n \log n)^{1 / 4}$.

Proof. We decompose $[0,1]^{2}$ into a subsquare $R_{1}$ and a moat $R_{2}:=$ $[0,1]^{2}-R_{1}$. We choose $R_{1}$ so that it has side length $1-C(\log n / n)^{1 / 2}$ and is centered within $[0,1]^{2}$. Let $\left|S_{r}^{\varepsilon}(n) \cap R_{1}\right|$ denote the sum of the lengths of the edges in $S_{r}^{\varepsilon}(n) \cap R_{1}$ and similarly for $\left|S_{r}^{\varepsilon}(n) \cap R_{2}\right|$. By Lemma 5.1 we have that $\Sigma^{\varepsilon}(n) \leq\left|S_{r}^{\varepsilon}(n) \cap R_{2}\right|$ with high probability. It will thus be enough to show

$$
\mathbb{E}\left|S_{r}^{\varepsilon}(n) \cap R_{2}\right| \leq C(n \log n)^{1 / 4} .
$$

Since $S_{r}^{\varepsilon}(n) \cap R_{1}$ is a feasible boundary triangulation of $\left\{U_{1}, \ldots, U_{n}\right\} \cap R_{1}$ with respect to $R_{1}$, we have

$$
\begin{aligned}
\left|S_{r}^{\varepsilon}(n)\right| & =\left|S_{r}^{\varepsilon}(n) \cap R_{1}\right|+\left|S_{r}^{\varepsilon}(n) \cap R_{2}\right| \\
& \geq \sigma_{r}\left(\left\{U_{1}, \ldots, U_{n}\right\} \cap R_{1}, R_{1}\right)+\left|S_{r}^{\varepsilon}(n) \cap R_{2}\right|,
\end{aligned}
$$

where the inequality follows by the minimality of $\sigma_{r}$.

The number of sample points in $R_{1}$ is a binomial random variable $\operatorname{Bi}(n, p)$ with parameters $n$ and $p, p:=$ area $R_{1}$. Taking expectations and scaling we get

$$
\mathbb{E}\left|S_{r}^{\varepsilon}(n)\right| \geq\left(1-C(\log n / n)^{1 / 2}\right) \mathbb{E} \sigma_{r}\left(\left\{U_{1}, \ldots, U_{\mathrm{Bi}(n, p)}\right\}\right)+\mathbb{E}\left|S_{r}^{\varepsilon}(n) \cap R_{2}\right| .
$$

By definition we have $\mathbb{E}\left|S_{r}^{\varepsilon}(n)\right| \leq \mathbb{E}\left|\sigma_{r}(n)\right|+\varepsilon$ and thus

$$
\begin{aligned}
\mathbb{E}\left|S_{r}^{\varepsilon}(n) \cap R_{2}\right| & \leq \mathbb{E} \sigma_{r}(n)+\varepsilon-\left(1-C(\log n / n)^{1 / 2}\right) \mathbb{E} \sigma_{r}(\operatorname{Bi}(n, p)) \\
& \leq \mathbb{E}\left(\sigma_{r}(n)-\sigma_{r}(\operatorname{Bi}(n, p))+\varepsilon+C(\log n / n)^{1 / 2} \mathbb{E}\left(\operatorname{Bi}(n, p)^{1 / 2}\right)\right.
\end{aligned}
$$

by the growth bound (3.2). By the continuity of $\sigma_{r}$, the above is bounded by

$$
\begin{aligned}
& \leq \mathbb{E}\left(|n-\operatorname{Bi}(n, p)|^{1 / 2}\right)+\varepsilon+C(\log n / n)^{1 / 2}(n p)^{1 / 2} \\
& \leq\left(\mathbb{E}(n-\operatorname{Bi}(n, p))^{1 / 2}+C(\log n)^{1 / 2}\right. \\
& =(n(1-p))^{1 / 2}+C(\log n)^{1 / 2} .
\end{aligned}
$$

Since $p:=\left(1-C(\log n / n)^{1 / 2}\right)^{2} \geq 1-C(\log n / n)^{1 / 2}$, we see that $n(1-p) \leq$ $C(n \log n)^{1 / 2}$, completing the proof of Lemma 5.2.

We are now in a position to establish the estimate (5.1) and thus conclude the proof of Theorem 1.2. 
Lemma 5.3. For all $0<\varepsilon<1$ we have $\sigma_{r}(n) \leq \sigma(n) \leq \sigma_{r}(n)+\Sigma^{\varepsilon}(n)+C$.

Clearly (5.1) follows from Lemma 5.3 since it is enough to take expectations and apply Lemma 5.2.

Proof. Let $0<\varepsilon<1$ and $S_{r}^{\varepsilon}(n)$ be as above. We claim that

$$
\sigma_{r}(n) \leq \sigma(n) \leq\left|S_{r}^{\varepsilon}(n)\right|+\Sigma^{\varepsilon}(n)+C .
$$

The first inequality follows by the definition of $\sigma_{r}$. To prove the second, we need to show that there is a feasible triangulation of $\left\{U, \ldots, U_{n}\right\}$ whose total length is bounded by $\left|S_{r}^{\varepsilon}(n)\right|+\Sigma^{\varepsilon}(n)+C$. Such a triangulation is obtained by triangulating the quadrilaterals and pentagons in the graph described by $S_{r}^{\varepsilon}(n)$ (recall that with high probability the graph contains no hexagons and at most four pentagons). We triangulate the quadrilaterals by adding their diagonals, the sum of whose lengths is at most the sum of $\Sigma^{\varepsilon}(n)$ and the perimeter of the unit square. A triangulation of the pentagons may be achieved with a cost bounded by a constant since there are at most four pentagons. Thus we have shown the claim. Since $\left|S_{r}^{\varepsilon}(n)\right| \leq \sigma_{r}(n)+\varepsilon$, the result follows.

We have now proved Theorem 1.2 and turn our attention to the proof of Theorem 1.3. The proof depends on the following general rate result, which is a slight modification of a previous result of Redmond and Yukich (1994), Theorem 1.2(b).

THEOREM 5.4 (Rates of convergence). Let L be a quasi-additive continuous Euclidean functional of order 1 on $[0,1]^{2}$ such that the following "add-one bound" is satisfied:

$$
\left|\mathbb{E} L\left(U_{1}, \ldots, U_{n}\right)-\mathbb{E} L\left(U_{1}, \ldots, U_{n+1}\right)\right| \leq C(\log n / n)^{1 / 2} .
$$

If $\left|\mathbb{E} L\left(U_{1}, \ldots, U_{n}\right)-\mathbb{E} L_{r}\left(U_{1}, \ldots, U_{n}\right)\right| \leq \alpha(n)$, where $\alpha(n)$ is a function of $n$, then

$$
\left|\mathbb{E} L\left(U_{1}, \ldots, U_{n}\right)-\beta(L) n^{1 / 2}\right| \leq C\left[\alpha(n) \vee(\log n)^{1 / 2}\right]
$$

The proof of this result essentially follows Redmond and Yukich (1994), where the same type of estimate is obtained when the function $\log n$ is replaced by a constant function.

Proof of Theorem 1.3. To apply Theorem 5.4 to triangulations, we must show that $\sigma$ satisfies the estimate (5.4). For all $\varepsilon>0$ and $U_{1}, \ldots, U_{n}$ we recall that $G_{\varepsilon}:=G_{\varepsilon}\left(U_{1}, \ldots, U_{n}\right)$ are the uniquely defined Steiner sets with the property that

$$
\left|S\left(\left\{U_{1}, \ldots, U_{n}\right\} \cup G_{\varepsilon}\right)\right| \leq \sigma\left(U_{1}, \ldots, U_{n}\right)+\varepsilon .
$$


Recall that $\Delta^{\varepsilon}\left(U_{1}, \ldots, U_{n}\right)$ denotes the collection of triangles generated by $S\left(\left\{U_{1}, \ldots, U_{n}\right\} \cup G_{\varepsilon}\right)$. Notice that

$$
\begin{aligned}
\sigma\left(U_{1}, \ldots, U_{n}\right) & \leq \sigma\left(U_{1}, \ldots, U_{n+1}\right) \\
& \leq\left|S\left(\left\{U_{1}, \ldots, U_{n}\right\} \cup G_{\varepsilon}\right)\right|+D(\varepsilon, n) \\
& \leq \sigma\left(U_{1}, \ldots, U_{n}\right)+D(\varepsilon, n)+\varepsilon,
\end{aligned}
$$

where $D(\varepsilon, n)$ is the diameter of the random triangle in $\Delta^{\varepsilon}\left(U_{1}, \ldots, U_{n}\right)$ which contains the point $U_{n+1}$. By Lemma 5.1 we have $D(\varepsilon, n) \leq C(\log n / n)^{1 / 2}$ with high probability where $C$ does not depend upon $\varepsilon$. Letting $\varepsilon=$ $(\log n / n)^{1 / 2}$ gives the high probability bound

$$
\left|\sigma\left(U_{1}, \ldots, U_{n}\right)-\sigma\left(U_{1}, \ldots, U_{n+1}\right)\right| \leq C(\log n / n)^{1 / 2}
$$

and therefore the add-one bound

$$
\left|\mathbb{E} \sigma\left(U_{1}, \ldots, U_{n}\right)-\mathbb{E} \sigma\left(U_{1}, \ldots, U_{n+1}\right)\right| \leq C(\log n / n)^{1 / 2} .
$$

Thus, by (5.1) and (5.5) we obtain

$$
\left|\mathbb{E} \sigma\left(U_{1}, \ldots, U_{n}\right)-\beta(\sigma) n^{1 / 2}\right| \leq C(n \log n)^{1 / 4}
$$

which is the desired estimate (1.8). This concludes the proof of Theorem 1.3.

6. The probabilistic Plateau functional. To establish Theorem 1.4 we may adapt the above approach to the three-dimensional setting. We fix $D$ once and for all, $1<D<\infty$. It is helpful first to enlarge the definition of $\tau:=\tau_{D}$ in the following way. Let $F \subset \Delta$ be a finite set, where $\Delta \subset[0,1]^{3}$ is a convex polyhedron. Given $D$, a tetrahedralization $T_{D}(F, \Delta)$ is a decomposition of $\Delta$ into tetrahedra with aspect ratios bounded by $D$ such that the tetrahedral vertices coincide with $F$ and the corners of $\Delta$. Analogously to $\tau_{D}(F)$, we define

$$
\tau_{D}(F, \Delta):=\inf _{G}\left|T_{D}(F \cup G, \Delta)\right|,
$$

where $\left|T_{D}(F \cup G, \Delta)\right|$ denotes the total surface area and where $G$ ranges over finite sets in $\Delta$.

We now consider the properties of the Plateau functional $\tau:=\tau_{D}$. Notice that $\tau(\alpha F)=\alpha^{2} \tau(F)$ and thus $\tau$ is homogeneous of order 2. We may check that $\tau$ is subadditive on $\mathbb{R}^{3}$ by following the arguments of Lemma 3.2. Also, for any $F \subset[0,1]^{3}$ we have $\tau(F) \leq C\{\operatorname{card} F\}^{1 / 3}$, which we may establish by following the induction arguments of Lemma 3.3 [here and in all that follows, $C:=C(D)$ denotes a constant depending only on $D$ and whose value may vary from line to line]. Indeed, for $n \geq 1$, set $\varphi(n):=\max \tau(F)$, where the max is over all choices of $F \subset[0,1]^{3}$ with card $F=n$. By decomposing $[0,1]^{3}$ into eight subcubes of edge length $1 / 2$, we obtain from subadditivity,

$$
\varphi(n) \leq \max \left\{\frac{1}{4} \sum_{i=1}^{8} \varphi\left(a_{i}\right): \sum_{i=1}^{8} a_{i}=n\right\},
$$


where $1 / 4$ represents the square of the scaling factor $1 / 2$. Since $\sum_{i=1}^{8} a_{i}^{1 / 3} \leq$ $4 n^{1 / 3}$ when $\sum_{i=1}^{8} a_{i}=n$, the desired bound $\varphi(n) \leq C n^{1 / 3}$ follows via induction.

To see that $\tau$ is a continuous subadditive Euclidean functional on $\mathbb{R}^{3}$ of order two, that is, satisfies conditions (1.1)-(1.4) with $d=3$ and $p=2$, it remains only to verify continuity

$$
|\tau(F \cup G)-\tau(F)| \leq C\{\operatorname{card} G\}^{1 / 3} .
$$

We follow the approach used to verify the continuity of $\sigma$.

For all $\varepsilon>0$ and $F \subset[0,1]^{3}$ let $G_{\varepsilon}:=G_{\varepsilon}(F)$ be Steiner sets such that

$$
\left|T_{D}\left(F \cup G_{\varepsilon}\right)\right| \leq \tau_{D}(F)+\varepsilon .
$$

Let $T^{\varepsilon}(F):=T_{D}\left(F \cup G_{\varepsilon}\right)$. Let $\Delta^{\varepsilon}(F)$ denote the collection of tetrahedra defined by $T^{\varepsilon}(F)$ and let $\mathscr{E}^{\varepsilon}:=\mathscr{E}^{\varepsilon}(F)$ denote the collection of tetrahedral edges. Since the tetrahedra have uniformly bounded aspect ratios, the sum of the cubes of the edges in $\mathscr{E}^{\varepsilon}$ is bounded by a finite constant $C, C:=C(D)$. Using the growth bound $\tau(F) \leq C\{\text { card } F\}^{1 / 3}$, we easily establish the analog of (3.7), namely

$$
\tau(F \cup G) \leq\left|T^{\varepsilon}(F)\right|+C \sum_{i=1}^{J}\left|E_{i}\right|^{2}\left\{\operatorname{card}\left(G \cap \Delta_{i}\right)\right\}^{1 / 3},
$$

where $E_{i}$ is the longest edge of tetrahedron $\Delta_{i}, \Delta_{i} \in \Delta^{\varepsilon}(F)$. Hölder's inequality, together with the bound $\sum_{i=1}^{J}\left|E_{i}\right|^{3} \leq C$, completes the proof of continuity.

Given a convex polyhedron $\Delta \subseteq[0,1]^{3}$ and $F \subset \mathbb{R}^{3}$ such that $\operatorname{co}(F) \supset \Delta$, we next define a "boundary tetrahedralization" of $\Delta$ induced by $F$ in the same way that we defined boundary triangulations. Given $1<D<\infty$ fixed, consider a $D$-tetrahedralization of $\operatorname{co}(F)$. Such a tetrahedralization partitions $\operatorname{co}(F)$ into tetrahedra with aspect ratios bounded by $D$ and whose vertices coincide with $F$. The $D$-tetrahedralization of $\operatorname{co}(F)$, having the property that the total surface area of the restriction of its graph to the interior of $\Delta$ is minimal, is called the "boundary $D$-tetrahedralization" of $\Delta$ induced by $F$ and is denoted by $T_{r, D}(F, \Delta)$. The boundary $D$-tetrahedralization of $\Delta$ thus generates the usual tetrahedra as well as polyhedra with faces contained in the boundary of $\Delta$. We let $\left|T_{r, D}(F)\right|$ denote the total surface area of the faces of the polyhedra in the interior of $\Delta$. We let

$$
\tau_{r, D}(F, \Delta):=\inf _{G}\left|T_{r, D}(F \cup G, \Delta)\right|,
$$

where $G$ ranges over all finite sets in $\mathbb{R}^{3}$ with the property that $\operatorname{co}(F \cup G) \supset \Delta$. We will suppress mention of $D$ and henceforth write $\tau_{r}(F)$ for $\tau_{r, D}\left(F,[0,1]^{3}\right)$ and $\tau_{r}(F, \Delta)$ instead of $\tau_{r, D}(F, \Delta)$.

Given this extended definition of $\tau_{r}$, observe that $\tau_{r}$ is a continuous superadditive Euclidean functional on $\mathbb{R}^{3}$ of order 2 ; that is, it satisfies conditions (1.1), (1.2), (1.3') and (1.4) with $p=2$ and $d=3$. To verify continuity

$$
\left|\tau_{r}\left(F \cup G,[0,1]^{3}\right)-\tau_{r}\left(F,[0,1]^{3}\right)\right| \leq C_{2}\{\operatorname{card} G\}^{1 / 3},
$$

we may follow the approach of Section 4 . 
We may also show closeness of the functionals $\tau$ and $\tau_{r}$ as given by (1.5) with $p=2$ and $d=3$ there. To do this, we will follow the approach of Section 5. For all $\varepsilon>0$ and $U_{1}, \ldots, U_{n}$, we let $G_{\varepsilon}\left(U_{1}, \ldots, U_{n}\right)$ be the uniquely defined Steiner sets such that

$$
\left|T_{r}\left(\left\{U_{1}, \ldots, U_{n}\right\} \cup G_{\varepsilon}\right)\right| \leq \tau_{r}\left(U_{1}, \ldots, U_{n}\right)+\varepsilon .
$$

Let $T_{r}^{\varepsilon}(n):=T_{r}^{\varepsilon}\left(\left\{U_{1}, \ldots, U_{n}\right\} \cup G_{\varepsilon}\right)$. Let $\Delta_{r}^{\varepsilon}$ denote the collection of tetrahedra generated by the tetrahedralization $T_{r}^{\varepsilon}(n)$. Let $\mathscr{E}^{\varepsilon}:=\mathscr{E}^{\varepsilon}\left(U_{1}, \ldots, U_{n}\right)$ be the collection of edges formed from the intersection of the edges in $\Delta_{r}^{\varepsilon}$ and the interior of $[0,1]^{3}$. Let $\mathscr{F}^{\varepsilon}:=\mathscr{F}^{\varepsilon}\left(U_{1}, \ldots, U_{n}\right)$ be the collection of faces formed by intersecting the faces of $\Delta_{r}^{\varepsilon}$ and the interior of $[0,1]^{3}$.

The following estimate (6.2) is the analog of (5.2); its proof follows the proof of (5.2) with small modifications. Note that (6.3) follows from (6.2) and the bounded aspect ratio property of the tetrahedra.

LEMMA 6.1. With high probability all edges $E \in \mathscr{E}^{\varepsilon}$ satisfy the length bound

$$
|E| \leq C(\log n / n)^{1 / 3}
$$

and all faces $F \in \mathscr{F}^{\varepsilon}$ satisfy the area bound

$$
\text { area } F \leq C(\log n / n)^{2 / 3}
$$

Consider the faces in $\mathscr{E}^{\varepsilon}$ which meet the boundary of $[0,1]^{3}$ and let $\Sigma^{\varepsilon}(n):=\Sigma^{\varepsilon}\left(U_{1}, \ldots, U_{n}\right)$ denote the sum of their areas. Exactly as in Lemma 5.2 , we may find a rough estimate for $\Sigma^{\varepsilon}(n)$.

Lemma 6.2. For all $0<\varepsilon<1$ we have $\mathbb{E} \Sigma^{\varepsilon}(n) \leq C n^{2 / 9}(\log n)^{1 / 9}$.

Proof. Following the proof of Lemma 5.2, decompose $[0,1]^{3}$ into a subcube $Q_{1}$ centered within $[0,1]^{3}$ and a moat $[0,1]^{3}-Q_{1}$. Let the edge length of $Q_{1}$ be $1-C(\log n / n)^{1 / 3}$. Let $\left|T_{r}^{\varepsilon}(n) \cap Q_{1}\right|$ denote the sum of the areas of the faces in $T_{r}^{\varepsilon}(n) \cap Q_{1}$ and similarly for $\left|T_{r}^{\varepsilon}(n) \cap Q_{2}\right|$. By Lemma 6.1 we have $\Sigma^{\varepsilon}(n) \leq\left|T_{r}^{\varepsilon}(n) \cap Q_{2}\right|$ with high probability. It will be enough to show that

$$
\mathbb{E}\left|T_{r}^{\varepsilon}(n) \cap Q_{2}\right| \leq C n^{2 / 9}(\log n)^{1 / 9} .
$$

Now as in the proof of Lemma 5.2 we have

$$
\left|T_{r}^{\varepsilon}(n)\right| \geq \tau_{r}\left(\left\{U_{1}, \ldots, U_{n}\right\} \cap Q_{1}, Q_{1}\right)+\left|T_{r}^{\varepsilon}(n) \cap Q_{2}\right|
$$

and taking expectations we get

$$
\mathbb{E}\left|T_{r}^{\varepsilon}(n)\right| \geq\left(1-C(\log n / n)^{1 / 3}\right)^{2} \mathbb{E} \tau_{r}\left(\left\{U_{1}, \ldots, U_{\mathrm{Bi}(n, p)}\right\}\right)+\mathbb{E}\left|T_{r}^{\varepsilon}(n) \cap Q_{2}\right|,
$$


where $\operatorname{Bi}(n, p)$ denotes a binomial random variable with parameters $n$ and $p:=$ volume of $Q_{1}=\left(1-C(\log n / n)^{1 / 3}\right)^{3}$. Thus by scaling and the growth bounds for $\tau_{r}$ we have

$$
\begin{aligned}
\mathbb{E}\left|T_{r}^{\varepsilon}(n) \cap Q_{2}\right| & \leq \mathbb{E} \tau_{r}(n)+\varepsilon-\left(1-C(\log n / n)^{1 / 3}\right)^{2} \mathbb{E} \tau_{r}(\operatorname{Bi}(n, p)) \\
& \leq\left(\mathbb{E}(n-\operatorname{Bi}(n, p))^{1 / 3}+\varepsilon+C(\log n / n)^{1 / 3}(n p)^{1 / 3}\right. \\
& \leq(n(1-p))^{1 / 3}+C(\log n)^{1 / 3} \\
& \leq\left(n C(\log n / n)^{1 / 3}\right)^{1 / 3}+C(\log n)^{1 / 3} \\
& \leq C n^{2 / 9}(\log n)^{1 / 9} .
\end{aligned}
$$

This completes the proof of Lemma 6.2.

Let $\tau(n):=\tau\left(U_{1}, \ldots, U_{n}\right)$. The following is the analog of Lemma 5.3.

Lemma 6.3. For all $0<\varepsilon<1$ we have $\tau_{r}(n) \leq \tau(n) \leq \tau_{r}(n)+C\left(\Sigma^{\varepsilon}(n)+1\right)$.

Proof. Let $0<\varepsilon<1$ and $T_{r}^{\varepsilon}(n)$ be as in (6.1). We claim that

$$
\tau_{r}(n) \leq \tau(n) \leq\left|T_{r}^{\varepsilon}(n)\right|+C\left(\Sigma^{\varepsilon}(n)+1\right) .
$$

The first inequality follows by monotonicity. To prove the second, we need to show that there is a feasible tetrahedralization of $\left\{U_{1}, \ldots, U_{n}\right\}$ whose total surface area is bounded by $\left|T_{r}^{\varepsilon}(n)\right|+C\left(\Sigma^{\varepsilon}(n)+1\right)$. We observe that the polyhedra given by $T_{r}^{\varepsilon}(n)$ which meet the boundary are convex and may be tetrahedralized at a cost bounded by a constant multiple of the sum of the areas of their faces. The combined areas of their faces is the sum of $\Sigma^{\varepsilon}(n)$ and the area of the boundary of $[0,1]^{3}$. This proves (6.4). Combining (6.4) and (6.1) we obtain Lemma 6.3.

Lemmas 6.2 and 6.3 establish that the Plateau functional $\tau$ and its boundary version $\tau_{r}$ are close in the sense that they satisfy (1.5) with $p=2$ and $d=3$. More precisely, we have shown that

$$
\left|\mathbb{E} \tau\left(U_{1}, \ldots, U_{n}\right)-\mathbb{E} \tau_{r}\left(U_{1}, \ldots, U_{n}\right)\right| \leq C n^{2 / 9}(\log n)^{1 / 9} .
$$

We have therefore shown that $\tau$ and $\tau_{r}$ satisfy all the conditions of Theorem 1.1 with $p=2$ and $d=3$. Theorem 1.4 follows as desired.

It is a simple matter to find rates of convergence for the mean of $\tau$. Since we are in dimension three we can avoid appealing to Theorem 5.4. The subadditivity of $\tau$ gives $\mathbb{E} \tau\left(U_{1}, \ldots, U_{n}\right) \geq \beta(\tau) n^{1 / 3}$ and the superadditivity of $\tau_{r}$ gives $\mathbb{E} \tau_{r}\left(U_{1}, \ldots, U_{n}\right) \leq \beta(\tau) n^{1 / 3}$. By $(6.5)$ we thus have the rate estimate

$$
\left|\mathbb{E} \tau\left(U_{1}, \ldots, U_{n}\right)-\beta(\tau) n^{1 / 3}\right| \leq C n^{2 / 9}(\log n)^{1 / 9} .
$$

Whether these rates are optimal is unclear. 
7. Concluding remarks. Using the theory of continuous subadditive and superadditive Euclidean functionals, we have provided the asymptotics for the minimal Steiner triangulation functional as well as its three-dimensional counterpart, the discrete Plateau functional. With regard to the former, we have extended the work of Steele (1982) under the regularity condition that the triangles have bounded aspect ratios. Whether one can remove or relax this condition remains open. Additional questions which merit investigation include: (1) Is there an analog of Theorems 1.2 and 1.4 for triangulation and tetrahedralization functionals which do not use Steiner points? (2) Are there asymptotics for triangulation and tetrahedralization functionals which are defined in terms of simplicial complexes?

Acknowledgments. It is a pleasure to thank Sungchul Lee for valuable remarks which led to an improved presentation. I also thank Garth Isaak and an anonymous referee for suggestions leading to an improved exposition as well as the present formulation of a boundary triangulation.

\section{REFERENCES}

Beardwood, J., Halton, J. H. and Hammersley, J. J. (1959). The shortest path through many points. Math. Proc. Cambridge Philos. Soc. 55 299-327.

Bern, M. and EpPSTEIN, D. (1992). Mesh generation and optimal triangulation. In Computing in Euclidean Geometry (F. R. Hwang and D.-Z. Du, eds.) World Scientific, Singapore.

Courant, R. and Schiffer, M. (1950). Dirichlet Principle, Conformal Mapping, and Minimal Surfaces. Interscience, New York.

Douglas. J. (1939). Minimal surfaces of higher topological structure. Ann. of Math. 40 205-298.

Preparata, F. P. and Shamos, M. I. (1985). Computational Geometry. Springer, New York.

REDMOND, C. and YuKICH, J. E. (1994). Limit theorems and rates of convergence for subadditive Euclidean functionals. Ann. Appl. Probab. 4 1057-1073.

Redmond, C. and YUKich, J. E. (1996). Asymptotics for Euclidean functionals with powerweighted edges. Stochastic Process. Appl. 61 289-304.

RheE, W. S. (1993). A matching problem and subadditive Euclidean functionals. Ann. Appl. Probab. 3 794-801.

SalzBerg, S., Delcher, A., Heath, D. and Kasif, S. (1991). Learning with a helpful teacher. In Proceedings of the 12th International Joint Conference on Artificial Intelligence.

STEELE, J. M. (1981). Subadditive Euclidean functionals and non-linear growth in geometric probability. Ann. Probab. 9 365-376.

Steele, J. M. (1982). Optimal triangulation of random samples in the plane. Ann. Probab. 10 $548-553$.

Steele, J. M. (1997). Probability Theory and Combinatorial Optimization. SIAM, Philadelphia.

YukICH, J. E. (1995). Asymptotics for the stochastic TSP with power weighted edges. Probab. Theory Related Fields 203-220.

Yukich, J. E. (1998). Probability Theory of Classical Euclidean Optimization Problems. Lecture Notes in Math. 1675. Springer, Berlin.

DePARTMENT OF MATHEMATICS LEHIGH UNIVERSITY

Bethlehem , Pennsylvania 18015

E-MAIL: jey0@lehigh.edu 